\title{
TREE SPECIES CLASSIFICATION BASED ON NEUTROSOPHIC LOGIC AND DEMPSTER-SHAFER THEORY
}

\author{
B. $\mathrm{Hu} *$, Q. Li \\ Dept. of Earth and Space Science and Engineering, York University, Toronto, ON, Canada - (baoxin, qian2018) @yorku.ca
}

Commission III, WG III/6

KEY WORDS: Tree species classification, Dempster-Shafer theory, LiDAR, Multispectral, Panchromatic, Neutrosophic logic

\begin{abstract}
:
The objective of this study was to explore the use of multi-source remotely sensed data for individual tree species. To achieve this, a neutrosophic logic-based method was developed for tree species classification using the combined spectral, textural and structural information derived from WorldView-2 (WV-2) multispectral bands, WV-2 panchromatic band, and LiDAR (Light Detection And Ranging)-derived canopy height model (CHM), respectively. The developed method was tested on the data obtained over the Keele campus, York University, Toronto Canada and the KNN (K Nearest Neighbour) classification method. Twenty-one spectral, three textural and three structural features were used to classify five species (Norway maple, honey locust, Austrian pine, blue spruce, and white spruce). For this study, 522 trees were used for training and 223 for testing. The overall classification accuracy obtained by the proposed method was 0.82 . It was significantly improved compared with the KNN (0.73), weighted KNN (0.76), and fuzzy KNN (0.75) methods. In addition, Dempster-Shafer (DS) theory was explored to perform information fusion at the decision level in comparison to that at the feature level. The accuracies obtained by the fusion at the decision level were generally lower than those at the feature level. Even though promising results based on the neutrosophic logic were obtained during this proof-concept stage, studies are underway to perform more tests with a large number of tree crowns and more species and exploit other classification methods, such as support vector machine.
\end{abstract}

\section{INTRODUCTION}

Trees are essential components of urban ecosystems and provide a wide range of environmental, ecological, social, cultural and economic benefits. Accurate tree species classification is therefore important to city planning, ecological management, and other urban studies (Iovan et al., 2008). The recent advance in remote sensing technologies makes a huge amount of data from different sensors, such as high spatial resolution imagery and high point density LiDAR (Light Detection And Ranging), readily available. The high spatial resolution data allow one to take the advantage of the spatial and structural features of individual tree crowns, in addition to the commonly used spectral features (Zhang and $\mathrm{Hu}$ 2012, Alonzo et al. 2014, Li et al., 2015, and Fang et al., 2018). Furthermore, considering individual tree crowns as the basic units provides a flexible platform to integrate information from different sources of data. It is relatively easier to register data at individual crown levels, rather than at the individual pixel level. Multi-source remotely sensed data also challenge researchers to develop effective methods to utilize fully all available information of individual tree crowns in species classification.

Data obtained from different remote sensors are redundant, since they cover the same area. They are also complementary, because different sensors measure different physical properties of individual tree canopies. In addition, information contained in individual data sources is often imprecision and uncertain. Fusion of redundant and complementary data provides a complete description of a given canopy, while reducing imprecision and uncertainty. Even though studies have showed the potential to combine features from multi-source remotely sensed data in improving tree species classification (Fassnacht et al. 2016), further investigation is required for a full understanding of the discriminant powers of individual data set and to seek an efficient and effective way to combine information from different sources. This was the focus of this study.

Fusion of data from several sensors usually occurs at either the feature or the decision level (Fassnacht et al, 2016). In a featurelevel fusion, the feature sets derived from multi-sensors are consolidated into a single feature set. Machine learning methods, such as support vector machine (SVM) and random forest (RF), are commonly used to classify of the species of interest based on the combined features. SVM and RF are generally shown to be more robust for classification with a large number of features, in comparison with traditional parametric methods, such as maximum likelihood classification (Maxwell et al., 2018). However, the high dimension in the feature space resulted from the feature fusion is likely to be a concern for the applications where the size of training samples is small (Maxwell et al., 2018). In addition, the features derived from different data courses are usually treated equally in SVM and RF, even though some of the data sources may be more reliable than others.

In contrary, in a decision-level fusion, each data source is analysed separately, and the uncertainty and imprecision associated with each data source in the decision-making is measured and considered in the fusion process. Many mathematical concepts or formalisms can be used to measure uncertainty and imprecision and perform decision fusion, such as probability (Smets, 1993), fuzzy logic (Lucas and Araabi, 1999) possibilities (Dubois, 1986) and evidence theory (Dempster, 1967 and Shafer, 1976). Among them, the Demspter-Shafer (DS) theory provides a mean to explicitly handle uncertain, imprecise, and ignorance, and it is commonly used in remote sensing applications. The DS theory, developed by Dempster (1967) and

* Corresponding author 
Shafer (1976), is considered as a generalization of the Bayesian theory. With the DS theory, both inaccuracy and uncertainty in decision-making are represented through the definition of belief and plausibility functions that are calculated from a mass function. For a given evident, a mass function indicates the degree of belief in a proposition (also called as a focal element), given a piece of evidence and the belief can be associated with a simple proposition (a single class) or a compound proposition (a union of classes). The most crucial step in the application of the DS theory is the determination of the mass function. The commonly used methods in defining a mass function are based on a probabilistic distribution, mostly a Gaussian distribution (Walley, 1987), and the fuzzy logic (Waltz and Buede, 1986). Compared with probability-based approaches, a fuzzy approach has the advantage in handling inaccurate values and thus improves the mass distribution assigned to a compound disposition (Germain et al., 2002). Both probability-based and fuzzy approaches have been used in remote sensing image classification (Jouan, Allard, 2004, Mora et al, 2011, and Aval et al, 2019). Even though promising results are reported, classification accuracies could be further improved by explicitly addressing the uncertainties associated with training samples and individual data sets in information fusion.

Most of the existing methods in information fusion for classification are based on supervised learning. As in any supervised learning method, the quality of training samples is critical. It is common that some training samples may be problematic. However, most of the methods treat each training sample equally. To improve classification accuracy, it is important to develop a strategy to evaluate the quality of each training sample and consider it in the supervised learning process. In this study, the quality of training samples was characterized based on the neutrosophic logic that was created by Smarandache (1999) and considered in the determination of the mass function. In neutrosophic logic, a logical variable is associated with three components, the degrees of truth, indeterminacy, and falsehood. It can be used to uncertainty and ambiguity in data and mathematical models. The neutrosophic logic has been employed mostly in the segmentation of remotely sensed imagery (Naveed et al., 2019). To the best of knowledge, it has not been used for tree species classification or broadly land cover classification. In this study, the discriminant power related to individual data source is also explicitly accounted for in the combination rule. The proposed strategies were tested using K Nearest Neighbor (KNN) classifier and data collected over the Keele campus of York University, Toronto, Ontario, Canada.

\section{STUDY AREA AND DATA USED}

The study area is located in the Keele campus of York University, Toronto, Canada $\left(43.7735^{\circ} \mathrm{N}, 79.5019^{\circ} \mathrm{W}\right)$. Several remotely sensed data over the campus are available for this study. They include the Worldview-2 (WV-2) imagery obtained on July 21, 2016, and airborne LiDAR data acquired in April 2015 with a point density of 10 points per square meters (Airborne Imaging, 2015). The WV-2 imagery included one panchromatic band with a spatial resolution of $0.4 \mathrm{~m}$ by $0.4 \mathrm{~m}$ and eight multispectral bands with a spatial resolution of $1.6 \mathrm{~m}$ by $1.6 \mathrm{~m}$. The WV-2 imagery was converted to surface reflectance. The LiDAR data were collected using a Leica ALS 70 LiDAR instrument at a flying height of 1300 meters with a Pulse Rate Frequency (PRF) of $400 \mathrm{kHz}$. The horizontal accuracy of the collected LiDAR data was $30 \mathrm{~cm}$ and a vertical accuracy (on flat hard surfaces) of 10 $\mathrm{cm}$. A digital elevation model (DEM) and digital surface model (DSM) with the same spatial resolution as the WV-2 panchromatic band were subsequently generated from the LiDAR data cloud. The canopy height model (CHM) was derived as the difference between the DSM and DEM, and then smoothed with a 3 by 3 Gaussian low-pass filter to eliminate noise. The ortho-rectification method in ENVI software was used to co-register the WV-2 imagery to the CHM via LiDAR intensity image and LiDAR-derived DSM. Tie points were manually selected, and they were at various elevations (on the ground or on buildings) and evenly distributed across the study area. The resulting co-registration accuracy was at 0.57 pixels based on 400 tie points. The false colour composite of the WV-2 imagery and the LiDAR CHM are shown in Figure 1 and Figure 2 , respectively

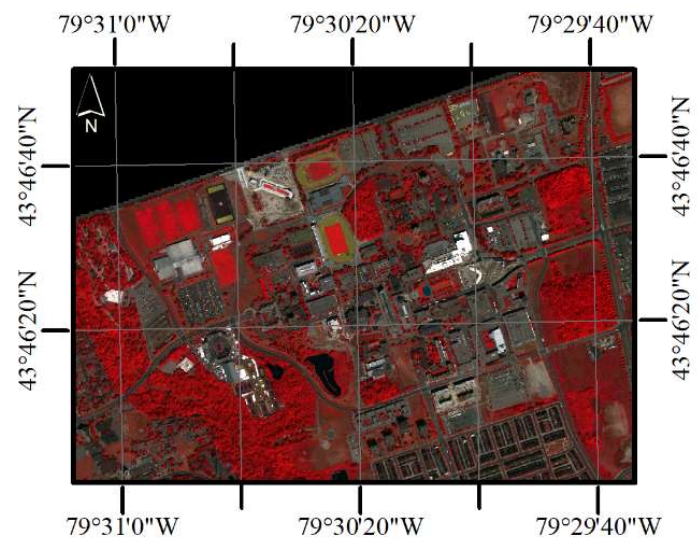

Figure 1: The false colour composite of the WV-2 imagery of the study area with the near-infrared band printed as red, red as green and green as blue, respectively.

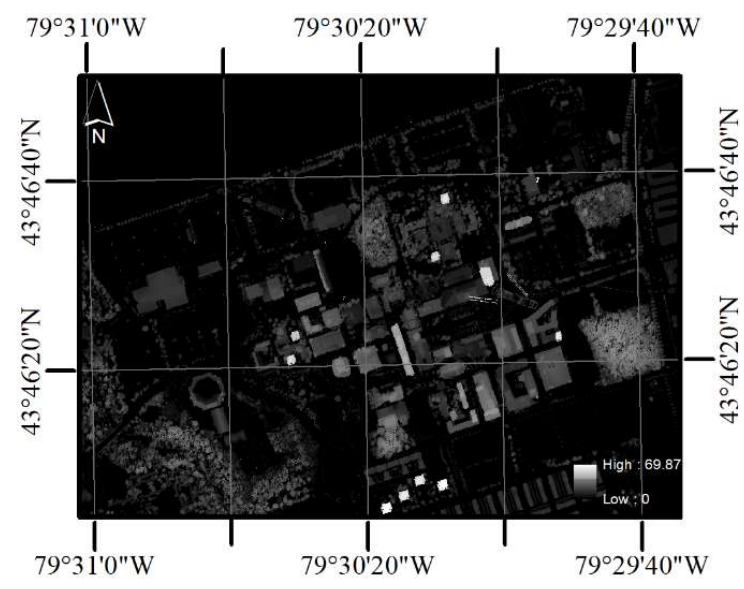

Figure 2: The CHM derived from the airborne LiDAR data over the study area.

There are trees of various species along roads and in woodlots. The six common species were selected for this study and they are Norway maple (Acer platanoides), honey locust (Gleditsia triacanthos), Austrian pine (Casuarina equisetifolia), blue spruce (Picea pungens), and white spruce (Picea glauca). A sample database of 745 trees was randomly selected based on street tree inventory surveyed by the Campus Services and Business Operations of York in June 2015. The selected tree samples were located along streets, near buildings and other high pedestrian areas, thereby representing the typical distribution of 
trees in an urban area. The sampled trees included 187 Norway maple, 180 honey locust, 159 Austrian pine, 120 blue spruce, and 99 white spruce. 522 of the 745 tree samples (around $70 \%$ ) were used for training and the rest (223 trees) for test. The crowns of these trees were delineated manually on the CHM by using information presented by both WV-2 imagery and LDAR data. An example of an individual tree crown appeared in these images is shown in Figure 3.
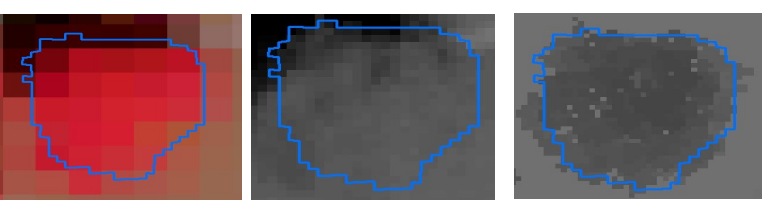

Figure 3: An individual tree crown appeared on the false colour image of the WV-2 multispectral bands (left), WV-2 panchromatic band (middle), and LDAR-derived CHM (right).

\section{METHOD}

\subsection{Feature extraction}

Three categories of features were derived for species classification, including 21 spectral features from the WV-2 multispectral bands, 3 textural features from the WV-2 panchromatic band, and 3 structural features from the LiDAR CHM image. These features were calculated for each tree crown.

The spectral features included the mean and standard deviation of the reflectance value in each of the eight spectral bands. In additional, five commonly used vegetation indices were generated to supplement the spectral information. As shown in Table 1, they were Normalized Difference Vegetation Index (NDVI), Enhanced Vegetation Index (EVI), Optimized Soil Adjusted Vegetation Index (OSAVI), Green Normalized Difference Vegetation Index (GNDVI), and Red Edge Normalized Difference Vegetation Index (RNDVI).

\begin{tabular}{|l|c|}
\hline Name & Equation \\
\hline NDVI & $\left(\rho_{\text {NIR } 1}-\rho_{\text {Red }}\right) /\left(\rho_{\text {NIR } 1}+\rho_{\text {Red }}\right)$ \\
\hline EVI & $1.6\left(\rho_{\text {NIR } 1}-\rho_{\text {Red }}\right) /\left(\rho_{\text {NIR } 1}+\rho_{\text {Red }}+0.16\right)$ \\
\hline OSAVI & $2.5\left(\rho_{\text {NIR1 }}-\rho_{\text {RED }}\right) /\left(\rho_{\text {NIR1 }}+6 \rho_{\text {Red }}-7.5\right.$ \\
& $\left.* \rho_{\text {Blue }}+1\right)$ \\
\hline GNDVI & $\left(\rho_{\text {NIR1 }}-\rho_{\text {Green }}\right) /\left(\rho_{\text {NIR } 1}+\rho_{\text {Green }}\right)$ \\
\hline RNDVI & $\left(\rho_{\text {RedEdge }}-\rho_{\text {Red }}\right) /\left(\rho_{\text {RedEdge }}+\rho_{\text {Red }}\right)$ \\
\hline
\end{tabular}

Table 1: Vegetation indices used for species classification. $\boldsymbol{\rho}$ refers to the reflectance and the subscript corresponds to the WV-2 band (Jensen, 2007)

The three texture features were derived from the WV-2 panchromatic band. The spatial arrangement of leaves and branches within tree crowns may be different for different tree species and it is likely to form the textural variation in grey tones of the panchromatic image. For instance, Norway maple exhibits a smooth and fine texture, while locust tree appears relatively coarser. In this study, three GLCM (gray-level co-occurrence matrix) measures, contrast, energy, and homogeneity were used (Haralick et al., 1973, and Ulaby et al., 1986).

Three structural features, the mean and standard deviation in height and area, were calculated to reflect the 3-D characteristics of tree species at the crown level. Even though age, surrounding condition and competition in urban environment all affect actual canopy height of trees, these derivatives predict structural arrangement of tree elements in a crown at both horizontal and longitudinal directions.

\subsection{Neutrosophic sets of training samples}

The neutrosophic logic was used, in this study, to explore the relationship of a given training sample to the five species classes. As described earlier, in the neutrosophic logic, the degrees of truth, indeterminacy, and falsehood are associated to a logical variable. The neutrosophic set of a training sample $i$ was defined as $\left(T_{i j}, I_{i}, F_{i}\right)$, where $T_{i j}$ was the degree of truth of train sample $i$ to species class $j$, and $I_{i}$ and $F_{i}$ were the degree of indeterminacy and falsehood of this training sample, respectively.

For a given category of features (spectral, textural, structural, or combined), denote the mean vector and variance and co-variance matrix of the species class $j$ as $\vec{m}_{j}$ and $\Sigma_{j}$ and the feature vector for a training sample $i$ as $\vec{x}_{i}$. The Mahalanobis distance from this sample to the centre of the species class $j$ was calculated (Equation (1)). Euclidean distance was also investigated, but the Mahalanobis distance performed better.

$$
d_{i j}^{2}=\left(\vec{x}_{i}-\vec{m}_{j}\right)^{T} \Sigma_{j}^{-1}\left(\vec{x}_{i}-\vec{m}_{j}\right)
$$

Inspired by the transformed divergency used in feature selection (Jensen, 2007), the distance between a training sample $i$ to the centre of a class $j$ was converted to a new measure $m_{i j}^{\prime}$ using Equation (2). The new measure was between 0 and 1 . The closer a sample was to a class, the larger this measure.

$$
m_{i j}^{\prime}=e^{-\left[\begin{array}{ll}
d_{i j}^{2}-\mathrm{mi} & \left(d_{j}^{2}\right)
\end{array}\right]}
$$

where $\quad \min \left(d_{j}^{2}\right)=$ the minimum distance of all training samples to the class $j$

Assume that the training sample $i$ belong to species class $k$. The neutrosophic set for this sample was calculated based on Equations (3).

$$
\begin{aligned}
& T_{i j}=m_{i j}^{\prime} \\
& I_{i}=m_{i l}^{\prime} \\
& F_{i}=\left\{\begin{array}{cc}
m_{i q}^{\prime} & q \neq k \\
1 & q=k
\end{array}\right.
\end{aligned}
$$

where $\quad m_{i l}^{\prime}=$ the largest measure from this sample to the centres of the species classes excluding class $k$ $m_{i q}^{\prime}=$ the smallest measure from this sample to the centres of the species classes

The membership measuring the likelihood of the training sample $i$ belonging to the species class $j$ was calculated using Equation (4).

$$
m_{i j}=\left\{\begin{array}{lc}
T_{i j} & m_{i k}^{\prime}>m_{i l}^{\prime} \\
T_{i j}\left(1-I_{i}\right) & m_{i k}^{\prime} \leq m_{i l}^{\prime} \\
0 & F_{i}=1
\end{array}\right.
$$

\subsection{KNN classification based on the neutrosophic logic}

KNN classifies an unknown tree crown based on the properties of its $\mathrm{K}$ closest training samples. In this study, the KNN classifier based on the neutrosophic logic (referred as neutrosophic KNN, hereafter) was implemented. The membership of an unknown tree crown $s$ associated to any species class $j$ was calculated using Equation (5). 


$$
m_{s j}=\frac{\sum_{i=1}^{K} w_{i} m_{i j}}{\sum_{i=1}^{K} w_{i}}
$$

where $\quad w_{i}=$ the inverse of the Euclidean distance between the crown $s$ and training sample $i$.

In this study, K was empirically determined as 10 . In addition, the weighted KNN (Dubani, 1976), and the fuzzy KNN (Keller et al., 1985) were also implemented, for comparison. With the same definition as in Equation (5), for the weighted $\mathrm{KNN}$, the membership of training sample $i$ to the species class to which it belonged was 1 and 0 for other classes. For the fuzzy KNN, the membership of training sample $i$ to the species class $j$ was defined as the inverse of the distance calculated by Equation (1).

\subsection{KNN classification based on the DS theory}

In the DS theory, a frame of discernment $\Theta$ is defined to represent all the classes under consideration: $\Theta=\left\{\theta_{1}, \theta_{2}, \ldots \theta_{N}\right\}$ and the power set $2^{\Theta}$ is then defined to include all the subset of $\Theta$ and the empty set $\phi$. The mass function within the range of $[0,1]$ is used to indicate the degree of belief in a hypothesis, given a piece of evidence. For each hypothesis in $2^{\Theta}, A_{i}$ (denoted as $A_{i} \subseteq 2^{\theta}$ ), the mass function $m\left(A_{i}\right)$ satisfies the following conditions:

$$
m\left(A_{i}\right) \in[0,1] ; m(\varnothing)=0 ; \sum_{A_{i} \subseteq 2^{\theta}} m\left(A_{i}\right)=1
$$

The aggregation of multiple belief functions from all of the available evidences is called Demspter's orthogonal sum or Demspter's rule of combination in the DS theory and it is calculated from evidences $(1,2, \ldots, n)$ in the following manner.

$$
m_{1,2, \ldots, n}(A)=\frac{\sum_{B_{1} \cap \ldots \cap B_{N}=A} \prod_{i=1}^{N} m_{i}\left(B_{i}\right)}{1-\sum_{B_{1} \cap \ldots \cap B_{N}=\varnothing} \prod_{i=1}^{N} m_{i}\left(B_{i}\right)}
$$

However, when the conflict among the evidences is larger, that is, the denominator in Equation (7) equals close to zero, the combination rule provides counter-intuition decision. To overcome this issue, different combination rules have been proposed. Among them is the Murphy's averaging rule (Murphy, 2000). In this study, we employed a weighted averaging rule and the weight was determined by using KNN classification. The memberships obtained from the KNN classifiers described in section 3.3 were used as mass functions.

\section{RESULTS AND DISCUSSION}

The overall classification accuracies for the weighted KNN, fuzzy KNN and neutrosophic KNN based on spectral, structural, and textual features individually and combined are shown in Table 2 .

\begin{tabular}{|l|l|l|l|l|}
\hline \multicolumn{2}{|c|}{} & \multicolumn{3}{|c|}{ KNN methods } \\
\cline { 3 - 5 } \multicolumn{2}{|c|}{ Features } & weighted & fuzzy & neutrosophic \\
\cline { 2 - 5 } & Structural & 0.70 & 0.61 & 0.67 \\
\cline { 2 - 5 } & Textural & 0.51 & 0.57 & 0.55 \\
\cline { 2 - 5 } & Combined & 0.76 & 0.38 & 0.37 \\
\hline
\end{tabular}

Table 2: Overall classification accuracies obtained using different KNN methods based on different features

As shown in Table 2, with individual features, classifications using the spectral features achieved the highest accuracies and those based on the textual features lowest. With all three KNN methods, classifications using the combined spectral, spectral, and textual features (i.e. information fusion at the feature level) achieved higher accuracies than those using individual features. The proposed neutrosophic KNN outperformed both weighted KNN and fuzzy KNN and obtained an overall accuracy of 0.82 using the combined features.

With the memberships of test samples belonging to each species class calculated based on individual features (spectral, structural and textural), the classification accuracies obtained by the information fusion at the decision level based on DS theory are shown in Table 3. From Table 3, it is clear that the spectral features had the highest the power in the discriminating the five species of interest and the lowest one was from the textural features. By combining these three groups of features using the proposed method, the classification accuracies were increased. The highest accuracy was achieved by using weighted KNN $(0.79)$ and the lowest accuracy was by fuzzy KNN (0.64). Compared with the results obtained by the fusion at the feature level (Table 2), one can see that for both fuzzy KNN and neutrosophic KNN, the feature level fusion outperformed the decision level fusion. On the contrary, the decision level fusion based on weighted KNN performed better than the fusion at the feature level. The low discriminant power by the textual features may complicate the results. Investigations are being undertaking to improve the classification accuracies by adding more textual features and by using more advanced classification methods.

\begin{tabular}{|l|l|l|l|}
\hline \multirow{2}{*}{ Spectral } & \multicolumn{3}{|c|}{ KNN methods } \\
\cline { 2 - 4 } & weighted & fuzzy & neutrosophic \\
\hline Structural & 0.70 & 0.61 & 0.67 \\
\hline Textural & 0.51 & 0.57 & 0.55 \\
\hline DS theory & 0.79 & 0.38 & 0.37 \\
\hline
\end{tabular}

Table 3: Overall classification accuracies obtained using different KNN methods based on information fusion at the decision level.

To further examine the classification results, the confusion matrices obtained by the neutrosophic $\mathrm{KNN}$ based on the fusion at the feature level and decision level are provided in Table 4 and Table 5, respectively. For both approaches, misclassification mostly occurred between blue spruce and white spruce. This might be due to the fact that these two species belong to the same genus and have similar properties. In addition, consistent good results were obtained for both Norway maple and honey locust.

\begin{tabular}{|c|c|c|c|c|c|c|c|}
\hline & \multicolumn{7}{|c|}{ Reference } \\
\hline ₹ี & & $\mathrm{MN}$ & LH & PA & SB & SW & $\begin{array}{l}\text { Producer's } \\
\text { accuracy }\end{array}$ \\
\hline : & $\mathrm{MN}$ & 48 & 2 & 0 & 0 & 0 & 0.96 \\
\hline 象 & LS & 7 & 47 & 1 & 5 & 2 & 0.75 \\
\hline s & PA & 0 & 0 & 43 & 1 & 3 & 0.91 \\
\hline$\overline{0}$ & SB & 0 & 2 & 4 & 24 & 4 & 0.71 \\
\hline & SW & 0 & 3 & 0 & 6 & 21 & 0.70 \\
\hline & $\begin{array}{l}\text { User's } \\
\text { accuracy }\end{array}$ & 0.87 & 0.87 & 0.89 & 0.67 & 0.70 & OA: 0.82 \\
\hline
\end{tabular}
The major discrepancy between the two different fusion approaches lied on the classifications of Austrian pine.

Table 4: The confusion matrix for the neutrosophic KNN using the combined features (feature level fusion), where MN, LS,

PA, SB, and SW stand for Norway maple, honey locust, Austrian pine, blue spruce, and white spruce, respectively. The overall accuracy (OA) is 0.82 . 


\begin{tabular}{|c|c|c|c|c|c|c|c|}
\hline & & & & Refer & & & \\
\hline$\Xi$ & & $\mathrm{MN}$ & LH & PA & SB & SW & $\begin{array}{l}\text { Producer's } \\
\text { accuracy }\end{array}$ \\
\hline . & $\mathrm{MN}$ & 47 & 1 & 0 & 0 & 0 & 0.98 \\
\hline 0 & LS & 8 & 45 & 7 & 3 & 0 & 0.71 \\
\hline $\bar{n}$ & PA & 0 & 0 & 30 & 0 & 0 & 1.0 \\
\hline$\overline{0}$ & SB & 0 & 4 & 7 & 25 & 5 & 0.61 \\
\hline & SW & 0 & 4 & 4 & 8 & 25 & 0.61 \\
\hline & $\begin{array}{l}\text { User's } \\
\text { accuracy }\end{array}$ & 0.85 & 0.83 & 0.63 & 0.69 & 0.83 & OA: 0.77 \\
\hline
\end{tabular}

Table 5: The confusion matrix for the neutrosophic KNN and DS theory, where MN, LS, PA, SB, and SW stand for Norway maple, honey locust, Austrian pine, blue spruce, and white spruce, respectively. The overall accuracy (OA) is 0.77 .

\section{CONCLUSIONS}

The information fusion methods developed based on the neutrosophic logic and DS theory for tree species classification was demonstrated to be promising based on the data obtained from WV-2 and airborne LiDAR data over the Keele campus, York University, Toronto Canada and the KNN classification method. Caution should be taken in that the classification tests were based on a limited number of species and training and testing samples, and further research with more species and field samples is needed. Furthermore, the KNN classification method was used due to its simplicity to prove concepts. Advanced classification methods, such as SVM, will be exploited in future works.

With the proposed method, spectral, textual, and structural features were derived from WV-2 multispectral bands, WV-2 panchromatic band, and LiDAR-derived CHM, respectively. For individual types of features, the spectral features were shown to be more effective in the discrimination of the five species of interest, namely, Norway maple, honey locust, Austrian pine, blue spruce, and white spruce. The textural features the least effective. An investigation is being carried out to derive advanced textural and structural features and they will be employed to improve the classification accuracy. The classification accuracy was improved by using multi-source remotely sensed data based on the fusion at both the feature and decision levels. For the cases tested, the feature level fusion performed better than the fusion at the decision level. For this study, the number of features from the combined spectral, structural, and textural remained small (27). The disadvantage of the fusion at the feature level might not evident. In future studies, more features will be used to evaluate the two fusion approaches.

The results also showed that the proposed neutrosophic logic $\mathrm{KNN}$ was outperformed the commonly used weighted KNN and fuzzy KNN and the overall classification accuracy reached 0.82 for the fusion at the feature level. Among the three KNN methods, the improvement using information fusion at the decision level was observed only for the weighted KNN, compared with the feature-level fusion. The advantage of the decision fusion was expected to be obvious for the cases with a large number of features and for combining the classifiers with reasonable good accuracies, in the comparison with information fusion at the feature level.

\section{ACKNOWLEDGEMENTS}

The authors would like to thank York University library and CSBO for the LiDAR and tree inventory data, respectively. We would like to acknowledge the financial support provided by the Natural Sciences and Engineering Research Council (NSERC) of Canada, Esri Canada, and Ontario Ministry of Agriculture, Food and Rural Affairs (OMAFRA). We would also like to thank Dr. Hui Li of York University and the Key Laboratory of Digital Earth Science, Aerospace Information Research Institute, Chinese Academy of Sciences for her help in the co-registration of the WV-2 imagery and LiDAR data.

\section{REFERENCES}

Alonzo, M., Bookhagen, B., and Roberts, D.A., 2014. Urban tree species mapping using hyperspectral and lidar data fusion. Remote Sensing of Environment, 148, 70-83. https://doi.org/10.1016/j.rse.2014.03.018

Aval, J., Fabre, S., Zenou, E., Sheeren, D., Mathieu, F., and Briottet, X., 2019. Object-based fusion for urban tree species classification from hyperspectral, panchromatic and nDSM data. International Journal of Remote Sensing, 40:14, 5339-5365, DOI: 10.1080/01431161.2019.1579937.

Dempster, A.P., 1967. Upper and lower probabilities induced by a multi-valued mapping. Annals of Mathematical Statistics, 38, 325-339.

Dubois, D., 1986. Belief structures: Possibility theory and decomposable confidence measures on finite sets. Comput. Artif. Intell. 5(5), 403-416.

Dudani, S.A. 1976. The distance-weighted k-nearest neighbour rule. IEEE Transactions on Systems, Man, and Cybernetics, SMC-6, 325-327.

Fang, F., McNeil, B.E., Warner, T.A., \& Maxwell, A.E., 2018. Combining high spatial resolution multitemporal satellite data with leaf-on LiDAR to enhance tree species discrimination at the crown level. International Journal of Remote Sensing, 39(23), 9054-9072. https://doi.org/10.1080/01431161.2018.1504343.

Fassnacht, F.E., Latifi, H., Stereńczak, K., Modzelewska, A., Lefsky, M., Waser, L.T., Straubf, C., Ghosh, A., 2016. Review of studies on tree species classification from remotely sensed data. Remote Sensing of Environment, 186, 64-87.

Germain, M., Voorons, M., Boucher, J.M., Bénié, G.B., 2002. Fuzzy statistical classification method for multiband image fusion. In: Proceedings of the Fifth International Conference on Information Fusion, 1, 178-184.

Haralick, M., Shanmugam, K., and Dinstein, I., 1973. Textural Features for Image Classification, IEEE Trans. on Systems, Man and Cybernetics, SMC-3, 610-621.

Iovan, C., Boldo, D., and Cord, M., 2008. Detection, characterization, and modelling vegetation in urban areas from high-resolution aerial imagery. IEEE Journal of Selected Topics in Applied Earth Observations and Remote Sensing Selected Topic, 1, 206-213.

Jenson, J. R., 2007. Remote Sensing of Environment: An Earth Resource Perspective. Second edition, Prentice-Hall. 
Jouan, A. and Allard, Y., 2004. Land use mapping with evidential fusion of features extracted from polarimetric synthetic aperture radar and hyperspectral imagery. Information Fusion 5, 251-267.

Keller, J.M., Gary, M.R., and Givens, J.A, 1985. A Fuzzy KNearest Neighbor Algorithm. IEEE Transactions on Systems, Man, and Cybernetics, SMC-15, 580-585.

Li, D., Ke, Y., Gong, H., and Li, X., 2015. Object-based urban tree species classification using bi-temporal worldview-2 and worldview-3 images. Remote Sensing, 7(12), 16917-16937.

Lucas, C. and Araabi, B.N., 1999. Generalization of the Dempster-Shafer theory: A fuzzy-valued measure. IEEE Trans. Fuzzy Syst. 7(3), 255-270.

Maxwell, A.E., Warner, T.A., and Fang, F., 2018. Implementation of machine-learning classification in remote sensing: An applied review. International Journal of Remote Sensing. https://doi.org/10.1080/01431161.2018.1433343.

Mora, B., Fournier, R., and Samuel, R., 2011. Application of evidential reasoning to improve the mapping of regenerating forest stands. International Journal of Applied Earth Observation and Geoinformation 13(3):458-467.

Murphy, C. K., 2000. Combining belief functions when evidence Conflicts. Decision support systems, 29 (1), 1-9.

Naveed, F., Hu, B., Wang, J. and Hall, B., 2019. Individual tree crown delineation using multispectral LiDAR data. Sensor, 19 (24), 5421.

Shafer G., 1976. A Mathematical Theory of Evidence, Princeton, NJ: Princeton University Press.

Smarandache, F.A, 1999. Unifying Field in Logics: Neutrosophic Logic. Neutrosophy, Neutroosphic Set, Neutrosophic Probability and Statistics, American Research Press, Rehoboth.

Smets, P., 1993. The disjunctive rule of combination and the generalized Bayesian theorem. Int. J. Approx. Reason. 9, 1-35.

Ulaby, F. T., Kouyate, F., Brisco, B., Williams, T., 1986. Textural information in SAR images. IEEE Transactions on Geoscience and Remote Sensing, 24 (2), 235-245.

Walley P., 1987. Belief function representation of statistical evidence. The annals of Statistics, 15 (4), 1439-1465.

Waltz E.L., and Buede D.M., 1986. Data fusion and decision support for command and control. IEEE Transactions on System, Man and Cybernetics, 16 (6), 865-879.

Zhang, K., and Hu, B., 2012. Individual urban tree species classification using very high spatial resolution airborne multispectral imagery using longitudinal profiles. Remote Sensing, $4(6), 1741-1757$. 